\title{
Radiological Features on HRCT and RT-PCR Testing for the Diagnosis of Coronavirus Disease 2019 (COVID-19) in China: A Comparative Study of 78 Cases in Pregnant Women
}

\author{
Ming Deng \\ Zhongnan Hospital of Wuhan University \\ Wenbo Sun \\ Zhongnan Hospital of Wuhan University \\ Jinxiang Hu \\ Zhongnan Hospital of Wuhan University \\ Liejun Mei \\ Zhongnan Hospital of Wuhan University

\section{Dinghu Weng} \\ Zhongnan Hospital of Wuhan University

\section{Bo Liu} \\ Hangzhou YITU Healthcare Technology Co., Ltd. \\ Haibo Xu ( $\nabla$ xuhaibo1120@hotmail.com) \\ Zhongnan Hospital of Wuhan University
}

\section{Research Article}

Keywords: Novel coronavirus pneumonia, Covid-19, SARS-CoV-2, Computed tomography, Pregnant women with COVID-19 pneumonia, Reverse transcription-polymerase chain reaction (RT-PCR)

Posted Date: April 6th, 2020

DOl: https://doi.org/10.21203/rs.3.rs-21005/v1

License: (a) This work is licensed under a Creative Commons Attribution 4.0 International License. Read Full License 


\section{Abstract}

Objective: We aimed to evaluate the quantitative parameters of CT scans performed on pregnant women with COVID-19 who had different reverse transcription-polymerase chain reaction (RT-PCR) results.

Methods: Pregnant women with suspected cases of COVID-19 pneumonia (confirmed by next-generation sequencing or RT-PCR) who underwent high-resolution lung CT scans were retrospectively enrolled. Patients were grouped based on the results of the RT-PCR and the first CT scan: group 1 (double positive patients; positive RT-PCR and CT scan) and group 2 (negative RT-PCR and positive CT scan). The imaging features and their distributions were extracted and compared between the two groups.

Results: Seventy-eight patients were admitted to the hospital between Dec 20, 2019, and Feb 29, 2020. The mean age of the patients was 31.82 years (SD 4.1, ranged from 21 to 46 years). The cohort included 14 (17.95\%) patients with a positive RT-PCR test and 64 (82.05\%) with a negative RT-PCR test, there were 37 (47.44\%) patients with a positive CT scan, and 41 (52.56\%) patients with a negative CT scan. The sensitivity, specificity, positive predictive value, negative predictive value and accuracy of CT-based diagnosis of COVID-19 were $85.71 \%, 60.94 \%, 32.40 \%, 95.12 \%$ and $65.38 \%$, respectively. COVID-19 pneumonia mainly involved the right lower lobe of the lung. There were 53 semi-quantitative and 59 quantitative parameters, which were compared between the two groups. There were no significant differences in the quantitative parameters. However, the Hellinger distance was significantly different between the two groups, albeit with a limited diagnostic value (AUC=0.63).

Conclusions: Pregnant women with pneumonia usually present with typical abnormal signs on CT. Although multidimensional CT quantitative parameters are somewhat different between groups of patients with different RT-PCR results, it is still impossible to accurately predict whether the RT-PCR will be positive, which would allow for the earlier detection of SARS-CoV-2 infection.

\section{Introduction}

In late December 2019, a number of cases of "unknown viral pneumonia" were reported in China, and were later determined to be caused by a novel coronavirus, severe acute respiratory syndrome coronavirus 2 (SARS-CoV-2) with highly infectious $(1,2)$. In the past three months, SARS-CoV-2 has become a global health threat(3-5).

In the context of the coronavirus disease 2019 (COVID-19) epidemic, pneumonia is a critical disease that threatens the health of pregnant women and fetuses $(6,7)$. COVID-19 can cause severe pneumonia, leading to respiratory failure, septic shock, multi-organ dysfunction syndrome, and even maternal death in a short period (8-10). In addition, the intrauterine safety of the fetus should be supervised in depth in pregnant women with COVID-19, with the aim of establishing an early diagnosis(11).

High-resolution CT (HRCT) can show millimeter-scale ground-glass opacities (GGOs), and it can play a crucial role in the periodic evaluation of COVID-19(12). However, HRCT cannot be synchronized with the 
results of reverse transcription-polymerase chain reaction (RT-PCR). This is also a challenge for clinicians, who can neither fully grasp the optimal sampling time for RT-PCR nor make accurate diagnoses in a timely manner $(13,14)$.

In this study, we focused on quantifying the radiological imaging characteristics of lesions compared with associated structures of the normal lung based on COVID-19 status. We used a rigorous artificial intelligence (Al) system to find the optimal imaging characteristics in a large cohort using HRCT images from patient who had confirmed RT-PCR results $(15,16)$. We aimed to explore the value of the quantitative parameters of multidimensional CT for predicting positive RT-PCR results. In addition, we sought to identify the problems that radiologists are most likely to overlook to enable patients to benefit from CT diagnosis.

\section{Methods}

\section{Study design and participants}

The Ethics Commission of our hospital approved this study (NO.2020-0037). Written informed consent was waived due to the nature of the emergency and the need for infection prevention. This single-center study was performed at a hospital designated to treat pregnant women with COVID-19 in Wuhan. We retrospectively analyzed patients from Dec 20, 2019, to Feb 28, 2020, who had been screened by RT- PCR, viral detection and CT examination, according to the interim guidance from the WHO and China Food and Drug Administration (CDFA). The Department of Medical Laboratory of our hospital performed the laboratory confirmation of SARS-CoV-2 and influenza virus infections. For inpatients who underwent at least one chest CT scan, their real-time RT-PCR results were confirmed within two days before or after the CT examination. If the first result was negative, we obtained throat swab specimens every other day during the next few days of hospitalization to clarify the result.

\section{Data collection and CT parameters}

We collected data on age, maternal history, symptoms (fever, cough, and dyspnea) and epidemic influenza history. Our nurses monitored the patient's temperature every day and checked for any of these symptoms. To obtain missing information, we consulted with family members by phone during and after the patient's hospital stay. When the pregnant women underwent lung CT examinations, we strictly protected other parts of the body and informed the patient of the radiation dose.

The CT instrument we used was one of two 64-slice spiral CT scanners: a GE Discovery 750HD scanner (GE Medical Systems, Milwaukee, WI) and a SOMATOM Definition (Siemens Healthineers, Forchheim, Germany). The patient was placed in a supine position in the middle of the examination bed, with arms raised and placed along the sides of the head. The CT scan was performed with a cross-sectional spiral scan ranging from the tip of the lung to the lower margin of the bilateral diaphragm. 
The scanning field of view was $40 \mathrm{~cm}$. The parameters of the CT scanner were as follows: detector collimation width $64 \times 0.625 \mathrm{~mm}$ and tube voltage $120 \mathrm{kV}$ (when the patient's BMI was $<25 \mathrm{~kg} / \mathrm{m} 2$, the tube voltage was $100 \mathrm{kV}$; when the patient's BMI was $\geq 25 \mathrm{~kg} / \mathrm{m} 2$, the tube voltage was $120 \mathrm{kV}$ ). The tube current (ranging from 100 to $350 \mathrm{mAs}$ ) was regulated by an automatic exposure control system (CARE Dose 4D, Siemens Healthineers) and slice automatic tube current modulation technique (GE Medical Systems). Images were reconstructed with a slice thickness of $1.25 \mathrm{~mm}$ and an interval of $1.0 \mathrm{~mm}$. Lung window images (window width: 1500 Hounsfield unit [HU], window level: - $700 \mathrm{HU}$ ) and mediastinal window images (window width: $200 \mathrm{HU}$, window level: $40 \mathrm{HU}$ ) were reconstructed.

\section{Image interpretation}

Images from our hospital were randomly assigned to two experts to independently interpret all cases without access to any clinical information, including age or sex. The two confirming radiologists had different levels of experience (MLJ [a senior radiologist with 35 years of experience] and WDH [a radiology resident with 5 years of experience in interpreting chest CT images]). Any controversies were handled by discussion and consensus.

For the quantitative diagnosis of pneumonia, Al software (Hangzhou YITU Healthcare Technology Co., Ltd.) for COVID-19 pneumonia was employed as the CT image analysis tool. The system combines a convolutional neural network and thresholding methods for segmentation of the left and right lungs and the detection of patchy shadows. The distribution of CT values in the lungs was calculated to obtain a histogram, and subsequently, the quantitative parameters were computed, including the lung volume, the inflammatory volume, and the proportion of total volume accounted for by the inflammatory volume.

We also evaluated the Hellinger distance (closely related to, although different from, the Bhattacharyya distance), which quantified the similarity between two probability distributions. We also introduced the Jaccard coefficient to compare the similarity and diversity of sample sets, which is defined as the ratio between the size of intersection and the size of union of two sets. If group 1 and group 2 are all coincident, then Jaccard coefficient equal to one is defined ( $\mathrm{J}$ range from 0 to 1 )

\section{Follow-up and additional cases}

Patients with initially negative RT-PCR results underwent a second RT-PCR test within 2-14days. If the patient underwent CT reexamination (due to the severity of the COVID-19 outbreak, we performed low radiation dose CT in all patients.), we performed before-and-after comparisons based on the results of the RT-PCR tests, and the images were manually reviewed by the abovementioned radiologists. Thereafter, we assigned the $\mathrm{CT}$ results to two radiologists for verification to ensure that the quantitative parameters could be observed on the CT images included in this study. Finally, according to the RT-PCR results, the CT data were divided into a positive group (Group 1) and a negative group (Group 2). 


\section{Statistical analysis}

Analyses were performed with SPSS software version 22.0. Normally distributed data are presented as the mean (SD), and standardized volume or CT value proportions are expressed as percentages (\%). RTPCR was used as the reference standard, and the sensitivity, specificity, positive predictive value (PPV), negative predictive value (NPV) and accuracy of CT were calculated. Before comparison, we carried out a homogeneity test of variance (F-test) between grouped data. Thereafter, the differences between the two groups were analyzed by independent sample $T$ tests (for 112 parameters extracted from CT images). The valuable parameters were analyzed using receiver operating characteristic (ROC) curves, and the areas under the curves (AUCs) were calculated.

\section{Results}

\section{General information and pregnancy epidemiology}

A total of 174 patients from the fever clinic and obstetrics department were included in this study. We excluded 84 outpatients and enrolled 90 pregnant women hospitalized for delivery. In addition, we excluded 12 patients with a history of pulmonary surgery, a history of infection, pleural effusion, and poor respiratory coordination. The final sample of 78 patients who had suspected cases of COVID-19 pneumonia were retrospectively enrolled in our study; the study selection process is shown in a flow chart (Figure 1).

Overall, the mean age was 31.82 years (SD 4.1; range 21-46y), and the mean gestational age was 38 weeks (SD $1.29 \mathrm{w}$; range $36-41 \mathrm{w})$. No significant difference in age $(P=0.448)$ was observed between groups. The most common symptoms in group 1 were fever (9/12, [75\%]) and dry cough (3/12, [25\%]). The common symptoms in group 2 were fever (11/25, [44\%]), dry cough (1/25, [4\%]), and flu symptoms $(4 / 25,[16 \%])$. The clinical information of the patients is summarized (Table 1$)$.

Epidemiologically, one patient was exposed to the hospital environment during her last perinatal examination. None of the patients had a history of exposure to the seafood market. In this study, we found that four patients had a history of human immunodeficiency virus (HIV) infection (Figure 2), one of them had an abnormal lung $\mathrm{CT}$, and three patients did not undergo CT examinations. Another four patients had a recent history of influenza virus infection with negative RT-PCR results, and no pulmonary CT abnormalities were found on the CT images.

\section{RT-PCR and CT results}

The results showed that $14(17.95 \%)$ patients had positive RT-PCR results, and $37(47.44 \%)$ patients had positive CT results; $12(85.71 \%)$ patients had positive results for both RT-PCR and CT. In all, 41(52.56\%) patients had negative CT and RT-PCR results. In addition, we found two (2/14) patients who had completely negative CT results but positive RT-PCR results (Table 1). According to the RT-PCR results, we 
calculated the diagnostic sensitivity, specificity, PPV, NPV and accuracy of CT, as shown in Table 2.

Finally, we summarized the characteristics of the two groups. There were 12 patients with positive RT-PCR and CT (Group 1) and 25 with negative RT-PCR and positive CT (Group 2). No significant differences in CT images were found between four patients with symptomatic influenza virus infections.

The clinical characteristics of the patients with positive CT results are summarized in Table 1.

Table 1: Characteristics of the 78 enrolled cases

\begin{tabular}{ll}
\hline Characteristics & \multicolumn{1}{c}{ Results } \\
\hline AgeIYears $\square$ & $31.82+4.1$ rage of 21 to 46 \\
Overall age & $27.1(21-30)$ \\
\hline 30 & $32.75(31-38)$ \\
\hline $30-40$ & $41.5(41-46)$ \\
\hline RT-PCR results & Number(percentage) \\
\hline Positive & $14(17.95 \%)$ \\
\hline Negative & $64(82.05 \%)$ \\
\hline CT results & $34(43.59 \%)$ \\
\hline Positive (mild) & $3(3.85 \%)$ \\
\hline Positive (severe) & $41(52.56 \%)$ \\
\hline Negative & Number(percentage) \\
\hline Other virus & $1(1.28 \%)$ \\
\hline HIV & $4(5.13 \%)$ \\
\hline Influenza virus &
\end{tabular}

Table 2: The performance of lung HRCT for COVID-19 according to the RT-PCR results

\begin{tabular}{|c|c|c|c|c|c|c|c|c|c|c|}
\hline Groups & $\begin{array}{l}\text { Total } \\
\text { patients }\end{array}$ & $\mathrm{TP}$ & $\mathrm{TN}$ & FP & FN & $\begin{array}{l}\text { Sensitivity } \\
\text { (\%) }\end{array}$ & $\begin{array}{l}\text { Specificity } \\
\text { (\%) }\end{array}$ & $\begin{array}{l}\text { PPV } \\
(\%)\end{array}$ & $\begin{array}{l}\text { NPV } \\
(\%)\end{array}$ & $\begin{array}{l}\text { Accuracy } \\
(\%)\end{array}$ \\
\hline Overall & 78 & 12 & 39 & 25 & 2 & 85.71 & 60.94 & 32.40 & 95.12 & 65.38 \\
\hline \multicolumn{11}{|c|}{ Age(years) } \\
\hline$\leq 30$ & 35 & 6 & 15 & 14 & 0 & 100 & 51.72 & 30 & 100 & 60 \\
\hline$>30$ & 43 & 6 & 24 & 11 & 2 & 75 & 68.57 & 35.29 & 92.31 & 69.77 \\
\hline
\end{tabular}




\section{CT quantitative and semi quantitative parameters}

The quantitative parameters included the volume $(\mathrm{mm} 3)$ and CT value $(\mathrm{HU})$ of the lesions, and there were no significant differences between the two groups (Table 3). Given the differences in lung volumes, we standardized the volumes of the lesions. Although the ratios of lesion volume to lung volume were calculated, no significant differences were found. The lesions were distributed in 5 lobes and 18 lung segments (bilateral distribution), and there were no significant differences in the volumes and proportions of lesions in each lung segment. However, there was an obvious trend towards larger volumes in the right lower lobe compared with the right upper lobe.

Table 3: Details of the inflammatory volume (IV) and proportion of the total lung volume accounted for by the inflammatory volume(POIV) 


\begin{tabular}{lccc}
\hline Parameters & Group1 & Group2 & P Value \\
\hline Inflammatory volume (cmI) & & \\
Whole lung & $79.1483 \pm 88.3214$ & $60.2052 \pm 65.746$ & 0.469 \\
Right lung & $40.5917 \pm 45.1150$ & $30.5832 \pm 43.601$ & 0.522 \\
Left lung & $38.5575 \pm 51.3670$ & $29.6216 \pm 45.479$ & 0.595 \\
Right upper lobe & $7.5683 \pm 15.0776$ & $5.2164 \pm 12.022$ & 0.611 \\
Right middle lobe & $0.6975 \pm 1.2360$ & $1.9932 \pm 6.2432$ & 0.484 \\
Right lower lobe & $32.3250 \pm 33.5777$ & $23.3728 \pm 37.600$ & 0.488 \\
Left upper lobe & $13.5217 \pm 18.2830$ & $11.2168 \pm 23.712$ & 0.769 \\
Left lower lobe & $25.0383 \pm 38.4856$ & $18.4044 \pm 32.255$ & 0.586 \\
Proportion of the inflammatory volume (\%) & & \\
Whole lung & $2.5650 \pm 3.5531$ & $2.0488 \pm 2.431$ & 0.607 \\
Right lung & $2.4183 \pm 3.0639$ & $1.8952 \pm 2.792$ & 0.608 \\
Left lung & $2.7567 \pm 4.5621$ & $2.3616 \pm 4.329$ & 0.799 \\
Right upper lobe & $1.2208 \pm 2.6214$ & $0.8292 \pm 1.819$ & 0.605 \\
Right middle lobe & $0.2408 \pm 0.4901$ & $0.7688 \pm 2.384$ & 0.298 \\
Right lower lobe & $4.6517 \pm 5.7531$ & $3.3336 \pm 5.633$ & 0.512 \\
Left upper lobe & $1.6083 \pm 2.2739$ & $1.7068 \pm 4.1195$ & 0.939 \\
Left lower lobe & $4.7000 \pm 9.4204$ & $3.7224 \pm 8.6072$ & 0.756 \\
\hline & & & \\
\hline
\end{tabular}

There were four main types of suspicious lesions distributed in the lung, which were accurately distinguished by quantitative CT values using histograms. The CT value ranges of GGOs, meshlike(Figure 3), mixed and solid lesions were -700 to $-600 \mathrm{HU},-600$ to $-500 \mathrm{HU},-500$ to $-200 \mathrm{HU}$ and -200 to $60 \mathrm{HU}$, respectively. Comparisons of the average, peak, and median CT values in the two groups showed no significant differences $(P>0.05)$. In addition, there were no significant differences in CT values among the pulmonary lobes and segments.

Using available big data regarding CT results for normal lungs as a reference, we found that the Hellinger distance $(\mathrm{t}=2.33, \mathrm{P}=0.015)$ and Jaccard coefficient $(\mathrm{t}=2.28, P=0.029)$ of the whole lung were significantly different between groups 1 and 2 . The Hellinger distance $(\mathrm{t}=2.23, P=0.032)$ and Jaccard coefficient $(\mathrm{t}=2.64, P=0.0124)$ in the right lobe were significantly different between the two groups. However, the 
Jaccard coefficient in the left lobe was not different between the two groups $(t=1.80, P=0.081)$. As shown in Table 4.

Table 4: The Hellinger distance and Jaccard coefficient in the two groups

\begin{tabular}{lccc}
\hline & Group1 & Group2 & P Value \\
\hline $\begin{array}{l}\text { Hellinger distance } \\
\text { Whole lung }\end{array}$ & $0.2117 \pm 0.0381$ & $0.2568 \pm 0.0694$ & 0.016 \\
\hline Left lung & $0.2158 \pm 0.0406$ & $0.2584 \pm 0.0754$ & 0.032 \\
\hline Right lung & $0.2100 \pm 0.0369$ & $0.2580 \pm 0.0664$ & 0.008 \\
\hline Jaccard coefficient & & & \\
\hline Whole lung & $0.7208 \pm 0.0730$ & $0.6384 \pm 0.1472$ & 0.029 \\
\hline Left lung & $0.7042 \pm 0.0810$ & $0.6340 \pm 0.1563$ & 0.081 \\
\hline Right lung & $0.7267 \pm 0.0649$ & $0.6376 \pm 0.1406$ & 0.012 \\
\hline
\end{tabular}

\section{ROC analysis of effective parameters}

The results of the ROC analysis showed that the Jaccard coefficient (AUC=0.63) had only limited diagnostic value, while the Hellinger distance $(A U C=0.313)$ had no diagnostic value. All the calculated data are listed in Table 5.

Table 5: ROC analysis of the Hellinger distance and Jaccard coefficient

\begin{tabular}{lcccc}
\hline & AUC P Value & \multicolumn{2}{c}{$95 \%$ CI } \\
\cline { 4 - 5 } & & & lower & upper \\
\hline $\begin{array}{c}\text { Hellinger distance } \\
\text { Whole lung }\end{array}$ & 0.277 & 0.03 & 0.103 & 0.45 \\
\hline Right lung & 0.4 & 0.34 & 0.19 & 0.609 \\
\hline Left lung & 0.313 & 0.069 & 0.131 & 0.495 \\
\hline Jaccard coefficient & & & & \\
\hline Whole lung & 0.63 & 0.206 & 0.453 & 0.807 \\
\hline Rght lung & 0.536 & 0.732 & 0.338 & 0.734 \\
\hline Left lung & 0.588 & 0.39 & 0.409 & 0.768 \\
\hline
\end{tabular}

Note: $95 \mathrm{CI}$ is $95 \%$ confidence interval. 


\section{Discussion}

Early diagnosis of COVID-19 is crucial for disease treatment and control(17). At present, the clinical value of CT for assessing COVID-19 is widely accepted(18). In our study, RT-PCR was used as the reference standard; the sensitivity, specificity and accuracy of CT were $85.71 \%, 60.91 \%$ and $65.38 \%$, respectively. The corresponding PPV and NPV were $32.43 \%$ and $95.12 \%$, respectively. However, our results are quite different from those reported in the literature(17). Our sensitivity was significantly lower $(85.71 \%$ vs $97 \%)$, while the specificity was higher ( $60.91 \%$ vs $25 \%$ ) than that reported in the literature. By comparing our results with those in the literature, we found that the main reason was that the majority of our patients had mild pneumonia, and the average age was significantly lower than that of the RT-PCR-positive patients in the literature. In this study, the PPV of CT was $95.12 \%$, which was also consistent with the explanations proffered above.In our study, there were still some patients with positive CT and negative RTPCR results $(19,20)$. We also noticed that the rate of positivity on RT-PCR was not high; this may be related to our sampling time, specimen source, and different stages of pneumonia(21).

Our results show that almost all RT-PCR-positive patients (12/14) had the characteristic CT features in the early phase, which were described in recent studies $(17,22-24)$. Despite the high sensitivity of CT, especially in patients younger than 30 years old (sensitivity is $100 \%$ ), there was still one patient with no symptoms and no abnormal manifestations on pulmonary CT (18). Even more surprisingly, a pregnant woman combined with HIV and COVID-19 (2).

Negative CT results cannot eliminate the possibility of infection with SARS-CoV-2, and CT manifestations suggesting infection with SARS-CoV-2 also do not immediately appear $(25,26)$; therefore, these patients may also simply be carriers. In addition, the inflammatory manifestations of COVID-19 on lung CT are very similar to those caused by HIV infection, which may lead to misdiagnosis and improper treatment. This indicates that a comprehensive and detailed history of viral infections can be very useful for the early detection of suspected cases.

As mentioned in previous studies, nearly $30 \%$ of patients infected by SARS-CoV-2 are women $(27,28)$. Among pregnant women, more than $90 \%$ had mild pneumonia, whose clinical histories were very similar. Therefore, whether qualitative CT parameters can accurately reflect the characteristics of pneumonia are become the key point. On the one hand, it is very difficult for radiologists to quantitatively measure lesions due to their different CT manifestations $(14,29)$. On the other hand, there may be overlap and subtle differences between pneumonia lesions that are difficult to identify accurately(12). Clinically, the volume, density and distribution of the manifestations are the most important parameters for clinicians (16). However, the results suggest our parameters were not significantly different between the groups with positive and negative RT-PCR results. This may be another indication of the diversity and complexity of COVID-19. In addition, the results suggested that pneumonia was more common in the dorsal segment of the lower lobe of the right lung, which was consistent with previous reports(22). The unexpected results suggested that the original belief that the larger the size of the lesions, the greater the number of lesions, and the wider the lung segment involved, the higher the probability was of positive RT-PCR detection was 
inaccurate. Other studies have suggested that a higher negative rate of throat swabs compared with sputum samples is a possible cause(19). In this study, samples were collected two or more times (2-8 times) from each patient to ensure the accuracy of the results as much as possible. These results still need to be confirmed by follow-up antibody testing for COVID-19.

In this study, we found two new valuable parameters, the Hellinger distance and Jaccard coefficient(30); Ernst Hellinger introduced the Hellinger distance in 1909(31). On the one hand, when we considered the whole lungs, we found that the Hellinger distance was similar between normal lungs and the lungs of patients with positive RT- PCR results, and the coincidence rate of Jaccard coefficient was relatively higher (72.67\%). These results were significantly different from those in patients with negative RT-PCR results. Obviously, the histogram analysis of each lesion indicated that there were no differences in the mean, peak and median CT values between the two groups. These two seemingly contradictory results suggest that the findings are similar whether pneumonia manifests as solid or mixed density lesions. However, the Hellinger distance indicated that the GGOs and fibrosis foci distributed in different positions along the curve were significantly different. This is consistent with the literature regarding the radiological characteristics of SARS-CoV-2 infection, such as GGOs and reticular opacities (18). These quantitative results strongly suggest that the pulmonary manifestations in patients with positive RT-PCR results are relatively more likely to be associated with GGOs and reticular opacities $(19,32)$. However, imaging changes may lag behind changes in patients' clinical symptoms, causing clinicians to miss the optimal timing for the RT-PCR.

The diagnostic value was based on a single figure of merit with a test AUCs of 0.63 for the Jaccard coefficient, while the AUC for Hellinger distance was 0.313. Based on these results, we cannot independently diagnose COVID-19 by using only the Hellinger distance and Jaccard coefficient (29).

Our study had several limitations. First, there is a limited window of time within which to determine the COVID-19 status of pregnant women before they give birth, and the number of cases that could be included was quite limited. Second, due to the limitations on CT radiation doses, it is difficult to perform follow-up CT reexaminations within a short period. Third, although we performed strict image quality control, there were several patients with small pulmonary nodules, which can affect feature extraction from CT images. Finally, other potential causes of CT imaging errors, such as the CT partial volume effect, could not be estimated.

In conclusion, the possibility of influenza virus and HIV infection should be given close attention in pregnant women. Despite the high PPV of CT examination, the limited value of CT for patients with mild pneumonia and no lesion cases. The current results do not support the prediction of COVID-19 pneumonia based on the parameters extracted from CT. Therefore, we should spend much more time investigating the value of CT rather than replacing RT-PCR with CT.

\section{Declarations}


Acknowledgments: We thank all our colleagues who helped us during the current study. This study was supported by grant 81771819 from the National Natural Science Foundation of China and grant 2017 YFC0108803 from the National Key Research and Development Plan of China.

Conflicts of interest statement and funding: We declare no competing interests.

\section{References}

1. Lee EYP, Ng MY, Khong PL. COVID-19 pneumonia: what has CT taught us? Lancet Infect Dis 2020

2. Bellani G, Laffey JG, Pham T, Fan E, Brochard L, Esteban A, et Epidemiology, Patterns of Care, and Mortality for Patients With Acute Respiratory Distress Syndrome in Intensive Care Units in 50 Countries. JAMA 2016;315:788-800

3. Hellewell J, Abbott S, Gimma A, Bosse NI, Jarvis Cl, Russell TW, et Feasibility of controlling COVID- 19 outbreaks by isolation of cases and contacts. Lancet Glob Health 2020

4. Fisher $D$, Wilder-Smith $A$. The global community needs to swiftly ramp up the response to contain COVID-19. Lancet 2020

5. Mahase E. Covid-19: WHO declares pandemic because of "alarming levels" of spread, severity, and inaction. BMJ 2020;368:m1036

6. Ji Y, Ma Z, Peppelenbosch MP, Pan Potential association between COVID-19 mortality and healthcare resource availability. Lancet Glob Health 2020

7. Chen Q, Liang M, Li Y, Guo J, Fei D, Wang L, et Mental health care for medical staff in China during the COVID-19 outbreak. Lancet Psychiatry 2020

8. Duan L, Zhu G. Psychological interventions for people affected by the COVID-19 epidemic. Lancet Psychiatry 2020

9. Rasmussen SA, Smulian JC, Lednicky JA, Wen TS, Jamieson Coronavirus Disease 2019 (COVID-19) and Pregnancy: What obstetricians need to know. Am J Obstet Gynecol 2020

10. Schwartz DA. An Analysis of 38 Pregnant Women with COVID-19, Their Newborn Infants, and Maternal-Fetal Transmission of SARS-CoV-2: Maternal Coronavirus Infections and Pregnancy Outcomes. Arch Pathol Lab Med 2020

11. Liu D, Li L, Wu X, Zheng D, Wang J, Yang L, et Pregnancy and Perinatal Outcomes of Women With Coronavirus Disease (COVID-19) Pneumonia: A Preliminary Analysis. AJR Am J Roentgenol 2020:1-6

12. Sun Q, Xu X, Xie J, Li J, Huang Evolution of Computed Tomography Manifestations in Five Patients Who Recovered from Coronavirus Disease 2019 (COVID-19) Pneumonia. Korean J Radiol 2020

13. Li K, Wu J, Wu F, Guo D, Chen L, Fang Z, et al. The Clinical and Chest CT Features Associated with Severe and Critical COVID-19 Pneumonia. Invest Radiol 2020

14. Xiong Y, Sun D, Liu Y, Fan Y, Zhao L, Li X, et al. Clinical and High-Resolution CT Features of the COVID- 19 Infection: Comparison of the Initial and Follow-up Changes. Invest Radio/ 2020 
15. Allam Z, Jones DS. On the Coronavirus (COVID-19) Outbreak and the Smart City Network: Universal Data Sharing Standards Coupled with Artificial Intelligence (Al) to Benefit Urban Health Monitoring and Management. Healthcare (Basel) 2020;8

16. Li L, Qin L, Xu Z, Yin Y, Wang X, Kong B, et al. Artificial Intelligence Distinguishes COVID-19 from Community Acquired Pneumonia on Chest Radiology 2020:200905

17. Ai T, Yang Z, Hou H, Zhan C, Chen C, Lv W, et al. Correlation of Chest CT and RT-PCR Testing in Coronavirus Disease 2019 (COVID-19) in China: A Report of 1014 Cases. Radiology 2020:200642

18. Zhao D, Yao F, Wang L, Zheng L, Gao Y, Ye J, et al. A comparative study on the clinical features of COVID-19 pneumonia to other pneumonias. Clin Infect Dis 2020

19. Chan JF, Yip CC, To KK, Tang TH, Wong SC, Leung KH, et al. Improved molecular diagnosis of COVID19 by the novel, highly sensitive and specific COVID-19-RdRp/Hel real-time reverse transcriptionpolymerase chain reaction assay validated in vitro and with clinical specimens. J Clin Microbio/ 2020

20. Hao W, Li M. Clinical diagnostic value of CT imaging in COVID-19 with multiple negative RT-PCR Travel Med Infect Dis 2020:101627

21. Lippi G, Simundic AM, Plebani M. Potential preanalytical and analytical vulnerabilities in the laboratory diagnosis of coronavirus disease 2019 (COVID-19). Clin Chem Lab Med 2020

22. Shi H, Han X, Jiang N, Cao Y, Alwalid O, Gu J, et Radiological findings from 81 patients with COVID19 pneumonia in Wuhan, China: a descriptive study. Lancet Infect Dis 2020

23. Bernheim A, Mei X, Huang M, Yang Y, Fayad ZA, Zhang N, et al. Chest CT Findings in Coronavirus Disease-19 (COVID-19): Relationship to Duration of Infection. Radiology 2020:200463

24. Fang Y, Zhang H, Xie J, Lin M, Ying L, Pang P, et al. Sensitivity of Chest CT for COVID-19: Comparison to RT-PCR. Radiology 2020:200432

25. Hu Z, Song C, Xu C, Jin G, Chen Y, Xu X, et al. Clinical characteristics of 24 asymptomatic infections with COVID-19 screened among close contacts in Nanjing, China. Sci China Life Sci 2020

26. Ji LN, Chao S, Wang YJ, Li XJ, Mu XD, Lin MG, et Clinical features of pediatric patients with COVID19: a report of two family cluster cases. World J Pediatr 2020

27. Chen S, Yang J, Yang W, Wang C, Barnighausen COVID-19 control in China during mass population movements at New Year. Lancet 2020

28. Gu X, Cao B, Wang J. Full spectrum of COVID-19 severity still being depicted - Authors' Lancet 2020

29. Liu Y, Balagurunathan Y, Atwater T, Antic S, Li Q, Walker RC, et Radiological Image Traits Predictive of Cancer Status in Pulmonary Nodules. Clin Cancer Res 2017;23:1442-1449

30. Chen CM, Huang YS, Fang PW, Liang CW, Chang A computer-aided diagnosis system for differentiation and delineation of malignant regions on whole-slide prostate histopathology image using spatial statistics and multidimensional DenseNet. Med Phys 2020;47:1021-1033

31. Yang GL, Le Cam, Lucien M. Asymptotics in Statistics: Some Basic Concepts. Berlin: Springer, 2000:

32. Yang W, Cao Q, Qin L, Wang X, Cheng Z, Pan A, et al. Clinical characteristics and imaging manifestations of the 2019 novel coronavirus disease (COVID-19):A multi-center study in Wenzhou 
Figures

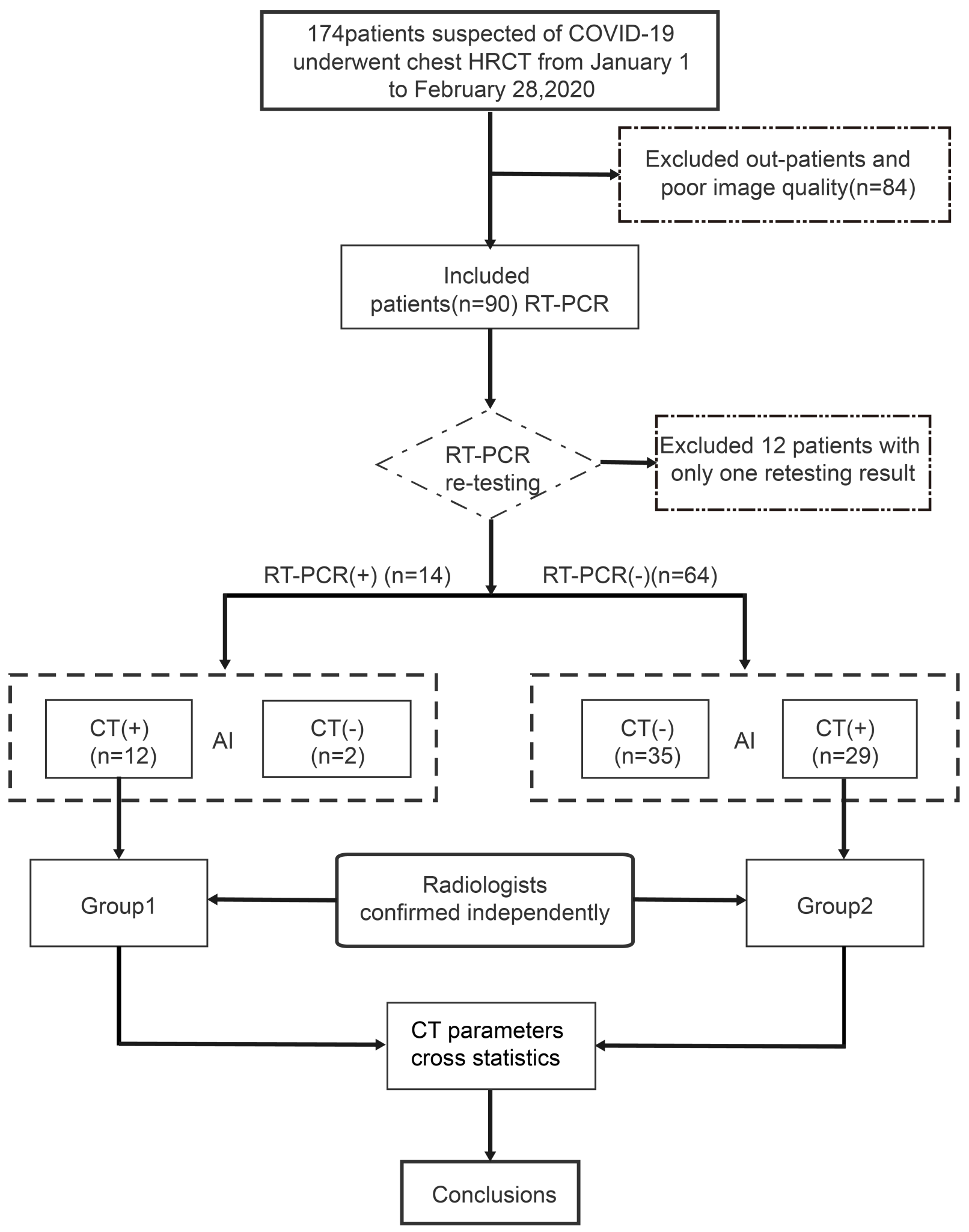

Figure 1

The flow chart of this study 

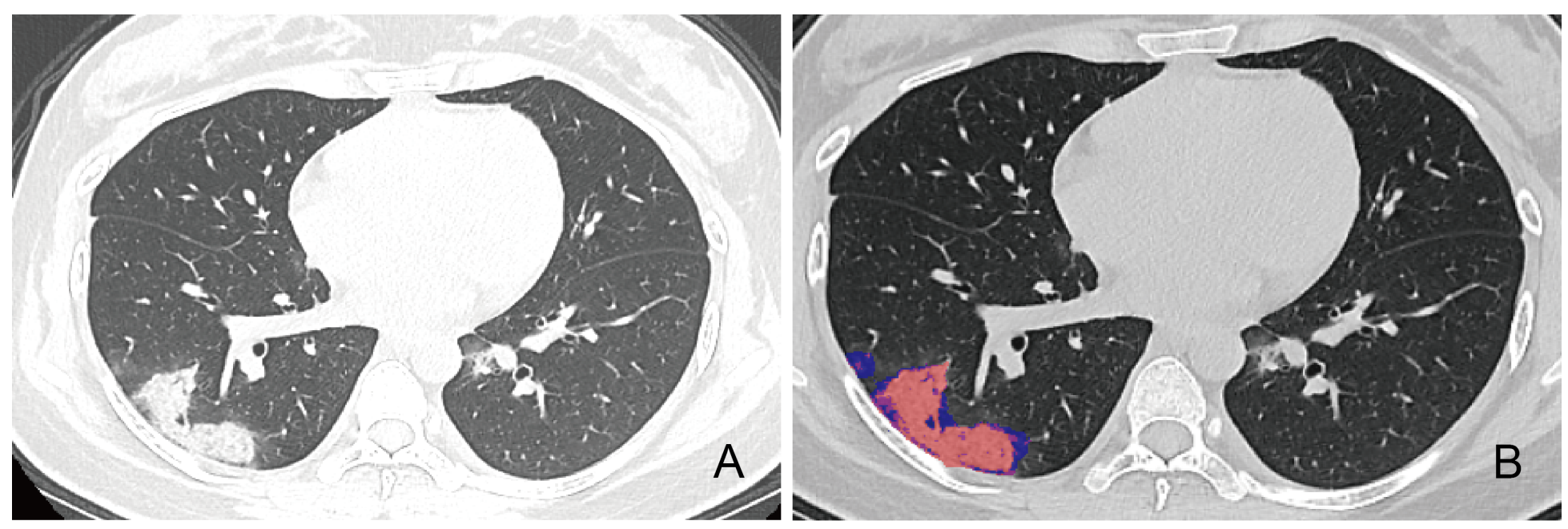

Figure 2

Chest CT images of a 27-year-old woman with fever for 2 days. The results of the RT-PCR assay for SARS-CoV-2 using a swab sample and the $C T$ results were positive $(A, B)$. The chest $C T$ findings show the ground-glass opacities and patchy consolidation. The final diagnosis was confirmed as COVID-19 combined with HIV (A). The CT color map shows the pattern of mixed lesions extracted by Al software (B). The Hellinger distance and Jaccard coefficient were 0.18 and 0.79 , respectively.
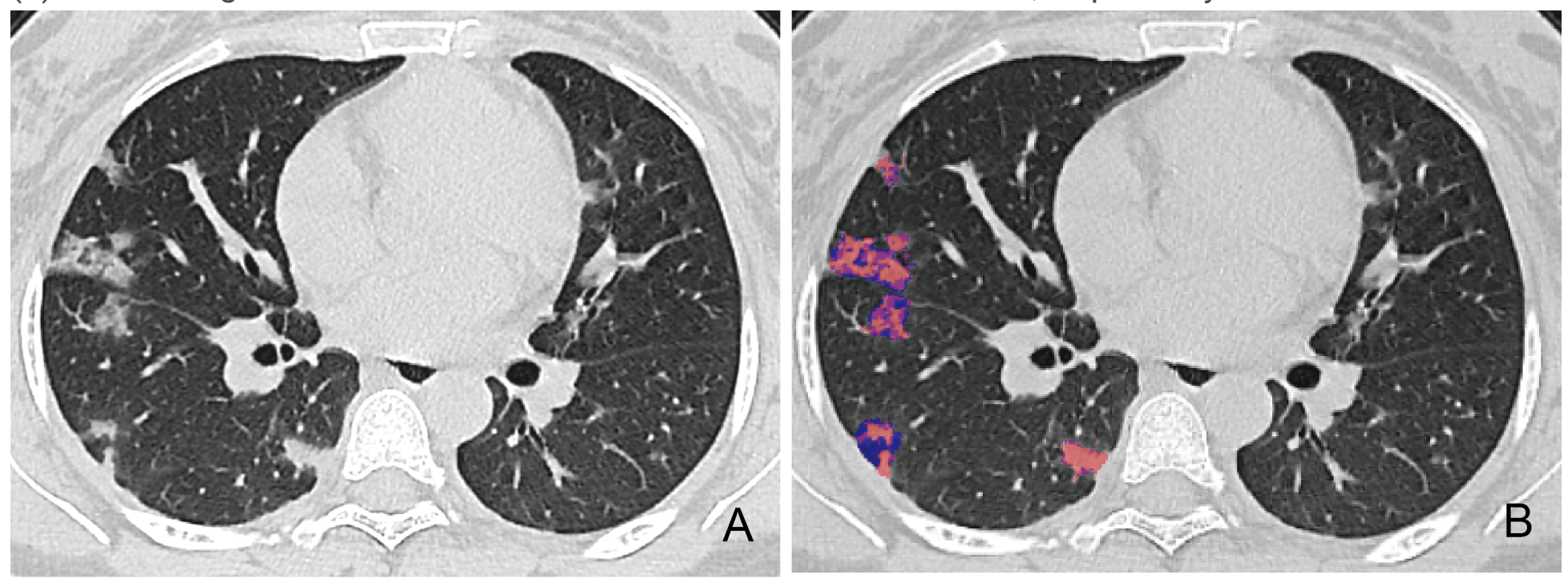

\section{Figure 3}

Chest CT images of a 26-year-old woman without any symptoms. A positive RT-PCR assay result for SARS-CoV-2 was obtained using a swab sample $(A, B)$. Chest $C T$ with an axial plane shows multiple manifestations with a focal ground-glass opacity (blue) and reticular opacities (brownish red) in the right lower lobe and middle lobe of right lung in the early stage of COVID-19 pneumonia (B). The Hellinger distance and Jaccard coefficient were 0.25 and 0.57 , respectively.

\section{Supplementary Files}


This is a list of supplementary files associated with this preprint. Click to download.

- SupplementaryTable1.docx 